\title{
ČECH SYSTEM DOES NOT INDUCE APPROXIMATE SYSTEMS
}

\author{
Vlasta Matijević
}

Dedicated to the memory of Professor Sibe Mardešić

\begin{abstract}
With every topological space $X$ is associated its Čech system $\boldsymbol{C}(X)=\left(|N(\mathcal{U})|,\left[p_{\mathcal{U V}}\right], \operatorname{Cov}(X)\right)$. It is well-known that the Cech system $C(X)$ of $X$ is an inverse system in the homotopy category $\mathrm{HPol}$ whose objects are polyhedra and morphisms are homotopy classes of continuous maps between polyhedra. We consider the following question posed by S. Mardešić. For a given Čech system $\left(|N(\mathcal{U})|,\left[p_{\mathcal{U}}\right], \operatorname{Cov}(X)\right)$ of a space $X$, is it possible to select a member $q_{\mathcal{U V}} \in\left[p_{\mathcal{U} \mathcal{V}}\right]$ in each homotopy class $\left[p_{\mathcal{U} \mathcal{V}}\right]$ in such a way that the obtained system $\left(|N(\mathcal{U})|, q_{\mathcal{U}}, \operatorname{Cov}(X)\right)$ is an approximate system? We answer the question in the negative by proving that for each Hausdorff arc-like continuum $X$ any such system $\left(|N(\mathcal{U})|, q_{\mathcal{U}}, \operatorname{Cov}(X)\right)$ is not an approximate system.
\end{abstract}

\section{INTRODUCTION AND MAIN RESULT}

Let $K$ be a simplicial complex. Denote by $|K|$ the carrier of $K$ (i.e. the union of all simplexes belonging to $K$ ) endowed with the $C W$-topology. By a polyhedron we mean a space $X$ such that $X=|K|$ for some simplicial complex $K$. If $X=|K|$ and $Y=|L|$ are polyhedra, then every simplicial map $f: K \rightarrow L$ determines in a natural way a continuous map $X \rightarrow Y$ for which we use the same notation $f$.

Recall that an inverse system in a category $\mathcal{C}$ is a collection $\boldsymbol{X}=\left(X_{\lambda}, p_{\lambda \lambda^{\prime}}, \Lambda\right)$ which consists of an index set $\Lambda$, endowed with a directed preorder $\preceq$, of objects $X_{\lambda}$ from $\mathcal{C}$, for $\lambda \in \Lambda$, and of morphisms $p_{\lambda \lambda^{\prime}}: X_{\lambda^{\prime}} \rightarrow X_{\lambda}$ from $\mathcal{C}$, for $\lambda \preceq \lambda^{\prime}$. On morphisms $p_{\lambda \lambda^{\prime}}$ one imposes the functorial requirement $p_{\lambda \lambda^{\prime}} p_{\lambda^{\prime} \lambda^{\prime \prime}}=p_{\lambda \lambda^{\prime \prime}}$, for $\lambda \preceq \lambda^{\prime} \preceq \lambda^{\prime \prime}$, and $p_{\lambda \lambda}=i d_{X_{\lambda}}$, for $\lambda \in \Lambda$.

2010 Mathematics Subject Classification. 54B35, 18B30, 54D30.

Key words and phrases. Inverse system, Čech system, approximate system, polyhedron, nerve of a covering, arc-like space, chainable space. 
With every topological space $X$ one associates an inverse system $\boldsymbol{C}(X)=\left(|N(\mathcal{U})|,\left[p_{\mathcal{U V}}\right], \operatorname{Cov}(X)\right)$ in the homotopy category HPol of polyhedra and homotopy classes of continuous maps called the Cech system of $X$. The index set $\operatorname{Cov}(X)$ is the set of all normal coverings $\mathcal{U}$ of $X$. A normal covering of $X$ is an open covering $\mathcal{U}$ which admits a partition of unity subordinated to $\mathcal{U}$. If $X$ is a paracompact space, then $\operatorname{Cov}(X)$ coincides with a set of all open coverings of $X$ (see [4, App. 1, §3.1 Corollary 1]). The set $\operatorname{Cov}(X)$ is preordered by the relation $\preceq$, where $\mathcal{U} \preceq \mathcal{V}$ means that $\mathcal{V}$ is a refinement of $\mathcal{U}$. For each $\mathcal{U} \in \operatorname{Cov}(X)$, a simplicial complex $N(\mathcal{U})$ is the nerve of $\mathcal{U}$ and $\left[p_{\mathcal{U V}}\right], \mathcal{U} \preceq \mathcal{V}$, is the unique homotopy class to which belong projections $p_{\mathcal{U V}}:|N(\mathcal{V})| \rightarrow|N(\mathcal{U})|$. Recall that vertices of $N(\mathcal{U})$ are the elements $U \in \mathcal{U}$, and vertices $U_{1}, \ldots, U_{n} \in \mathcal{U}$ span a simplex of $N(\mathcal{U})$ whenever $U_{1} \cap \cdots \cap U_{n} \neq \emptyset$. A projection $p_{\mathcal{U V}}:|N(\mathcal{V})| \rightarrow|N(\mathcal{U})|, \mathcal{U} \preceq \mathcal{V}$, is a continuous map determined by a simplicial map $p_{\mathcal{U}}: N(\mathcal{V}) \rightarrow N(\mathcal{U})$ which sends a vertex $V$ of $N(\mathcal{V})$ to a vertex $U$ of $N(\mathcal{U})$ with $V \subseteq U$. Any two projections $p_{\mathcal{U V}}, q_{\mathcal{U V}}:|N(\mathcal{V})| \rightarrow|N(\mathcal{U})|, \mathcal{U} \preceq \mathcal{V}$, are contiguous and thus also homotopic. Hence, projections $p_{\mathcal{U V}}:|N(\mathcal{V})| \rightarrow|N(\mathcal{U})|$ are not unique but they all belong to the same homotopy class. The Cech system is studied in detail in $[7$, App. $1, \S 3]$.

It was noticed long ago, in fifties of the past century, that studying compact Hausdorff non-metrizable spaces using inverse systems of polyhedra and their limits has some deficiencies. For instance, S. Mardešić proved that there exist 1-dimensional compact Hausdorff spaces which are not limits of inverse systems of 1-dimensional polyhedra ([2, Theorem 4]) and there exist chainable spaces which are not limits of inverse systems of arcs ([1, Theorem 6]). These results were among the reasons which led S. Mardešić and L.R. Rubin to introduce in 1989 a more flexible kind of inverse systems of metric compacta and continuous maps, called approximate inverse systems ([3]). S. Mardešić and T. Watanabe soon extended the notion to arbitrary topological spaces $([5])$. The main idea was to abandon the rigid functorial requirement $p_{\lambda \lambda^{\prime}} p_{\lambda^{\prime} \lambda^{\prime \prime}}=p_{\lambda \lambda^{\prime \prime}}$, for $\lambda \preceq \lambda^{\prime} \preceq \lambda^{\prime \prime}$, and allow the continuous maps $p_{\lambda \lambda^{\prime}} p_{\lambda^{\prime} \lambda^{\prime \prime}}$ and $p_{\lambda \lambda^{\prime \prime}}$ to differ. However, the difference should be arbitrarily small when $\lambda^{\prime}$ is sufficiently large. Precisely, an approximate inverse system (approximate system, for short) $\boldsymbol{X}=\left(X_{\lambda}, p_{\lambda \lambda^{\prime}}, \Lambda\right)$ consists of the same data as ordinary inverse system in the category Top of topological spaces and continuous maps. However, besides the requirement that $p_{\lambda \lambda}$ is the identity map, one imposes the following condition.

(A) For any $\lambda \in \Lambda$ and any normal covering $\mathcal{U}$ of $X_{\lambda}$, there exists an $\lambda^{\prime} \succeq \lambda$ such that for any $\lambda_{2} \succeq \lambda_{1} \succeq \lambda^{\prime}$ the maps $p_{\lambda \lambda_{1}} p_{\lambda_{1} \lambda_{2}}$ and $p_{\lambda \lambda_{2}}$ are $\mathcal{U}$-near, i.e. for each $x \in X_{\lambda_{2}}$ there exists a $U \in \mathcal{U}$ such that points $p_{\lambda \lambda_{1}} p_{\lambda_{1} \lambda_{2}}(x)$ and $p_{\lambda \lambda_{2}}(x)$ belong to $U$. 
S. Mardešić asked the following related question:

Let $\boldsymbol{C}(X)=\left(|N(\mathcal{U})|,\left[p_{\mathcal{U V}}\right], \operatorname{Cov}(X)\right)$ be the Čech system of a space $X$. Is it possible to select one projection $q_{\mathcal{U V}}$ in each homotopy class $\left[p_{\mathcal{U V}}\right], \mathcal{U} \preceq \mathcal{V}$, in such a way that the obtained system $\left(|N(\mathcal{U})|, q_{\mathcal{U V}}, \operatorname{Cov}(X)\right)$ is an approximate system? In other words, does the Čech system of a space $X$ induce approximate systems (associated with $X$ )?

We answer the question in the negative by showing that the Čech system $C(X)$ of an arbitrary Hausdorff arc-like space $X$ does not induce any associated approximate system as it is proved in Theorem 2.13 in the next section.

\section{ARC-LiKe SPACES AND Their ČECH SYSTEMS}

DEFINITION 2.1. Let $\mathcal{P}$ be a non-empty class of compact polyhedra and let $X$ be a $T_{1}$-space. We say that $X$ is $\mathcal{P}$-like, if for each open covering $\mathcal{U}$ of $X$, there exist a polyhedron $P \in \mathcal{P}$, an open covering $\mathcal{V}$ of $P$ and a surjective map $f: X \rightarrow P$ such that the open covering $f^{-1} \mathcal{V}=\left(f^{-1}(V), V \in \mathcal{V}\right)$ of $X$ refines $\mathcal{U}$.

Proposition 2.2. Let $\mathcal{P}$ be a non-empty class of compact polyhedra and let a $T_{1}$-space $X$ be $\mathcal{P}$-like. Then $X$ is a compact Hausdorff space. Furthermore, if each member of $\mathcal{P}$ is connected, then $X$ is connected, too.

Proof. Let $\mathcal{U}$ be an arbitrary open covering of $X$. Since $X$ is $\mathcal{P}$-like there exist a compact polyhedron $P$, a surjective map $f: X \rightarrow P$ and a finite open covering $\mathcal{V}$ of $P$ such that $f^{-1} \mathcal{V} \preceq \mathcal{U}$. Hence, $f^{-1} \mathcal{V}$ is a finite, open refinement of $\mathcal{U}$, which proves that $X$ is compact. Let $x, y \in X$ be different points of $X$. Since $X$ is a $T_{1}$-space, a collection $\mathcal{U}=(X \backslash\{x\}, X \backslash\{y\})$ is an open covering of $X$. Then, there exist a compact polyhedron $P$, a map $f: X \rightarrow P$ and an open covering $\mathcal{V}$ of $P$ such that $f^{-1} \mathcal{V} \preceq \mathcal{U}$. Note that $f(x) \neq f(y)$. Indeed, assume the contrary, i.e.. $f(x)=f(y)=p \in P$ and take an open set $V \in \mathcal{V}$ which contains $p$. Then $f^{-1} V$ is contained in $X \backslash\{x\}$ or in $X \backslash\{y\}$. However, $f^{-1} V$ contains $x$ and $y$ and we get a contradiction in both cases. Hence, $f(x) \neq f(y)$. Polyhedra are Hausdorff spaces and we can find open disjoint sets $W_{1}, W_{2} \subseteq P$ such that $f(x) \in W_{1}$ and $f(y) \in W_{2}$. Then $f^{-1} W_{1}$ and $f^{-1} W_{2}$ are required disjoint open neighborhoods of $x$ and $y$ respectively. Assume that each member of $\mathcal{P}$ is a connected polyhedron. We claim that $X$ is connected. Assume the contrary. Since $X$ is disconnected, there exist two non-empty disjoint open sets $U_{1}, U_{2}$ in $X$ such that $X=U_{1} \cup U_{2}$. Then $\mathcal{U}=\left(U_{1}, U_{2}\right)$ is an open covering of $X$ and there exist a compact connected polyhedron $P \in \mathcal{P}$, a map $f: X \rightarrow P$ and an open covering $\mathcal{V}$ of $P$ such that $f^{-1} \mathcal{V} \preceq \mathcal{U}$. Denote by $W_{1}=\cup\left\{V \in \mathcal{V}: f^{-1} V \subseteq U_{1}\right\}$ and by $W_{2}=\cup\left\{V \in \mathcal{V}: f^{-1} V \subseteq U_{2}\right\}$. First note that both open sets $W_{1}$ and $W_{2}$ are non-empty. Assume that $W_{1}=\emptyset$ and choose $x \in U_{1}$. Take $V \in \mathcal{V}$ such that $f(x) \in V$. Since $W_{1}=\emptyset$, it follows that $f^{-1} V \subseteq U_{2}$ and consequently $x \in U_{2}$ which is a contradiction. Also note 
that $W_{1}$ and $W_{2}$ are disjoint sets. Assume that there exist $p \in W_{1} \cap W_{2}$. Since $f$ is surjective, there exists $x \in X$ such that $f(x)=p$. Then there exists $V, V^{\prime} \in \mathcal{V}$ such that $p \in V, V^{\prime}, f^{-1} V \subseteq U_{1}$ and $f^{-1} V^{\prime} \subseteq U_{2}$. This implies $x \in U_{1} \cap U_{2}$ and we get a contradiction. So, $W_{1}, W_{2}$ are non-empty disjoint open sets and $P=W_{1} \cup W_{2}$. We conclude that $P$ is disconnected and get a contradiction.

Definition 2.3. A Hausdorff space $X$ is said to be arc-like (or snakelike), if $X$ is $\mathcal{P}$-like, where $\mathcal{P}$ consists only of the unit segment $I=[0,1] \subseteq \mathbb{R}$.

Here the unit segment $I=[0,1] \subseteq \mathbb{R}$ is considered as the carrier of a simplicial complex $K$ which has two vertices (points 0 and 1 ) and one 1-simplex. According to the previous proposition any arc-like space is a Hausdorff continuum, i.e. a compact connected Hausdorff space. Arc-like spaces can be characterized by a certain property of their open coverings. To show that firstly we define chainable coverings of a space.

Definition 2.4. A finite open covering $\mathcal{U}=\left(U_{i}, i=1, \ldots, n\right)$ of a space $X$ is called chainable provided $U_{i} \cap U_{j} \neq \emptyset$ if and only if $|i-j| \leq 1, i, j \in$ $\{1, \ldots, n\}$.

A polyhedron homeomorphic to the unit segment $I=[0,1] \subseteq \mathbb{R}$ is called an arc. Note that the nerve $|N(\mathcal{U})|$ of any chainable covering $\mathcal{U}=\left(U_{i}, i=\right.$ $1, \ldots, n), n \geq 2$, is an arc. If $\mathcal{V}$ is a chainable covering of a space $Y$ and $f: X \rightarrow Y$ is a surjective map, then $f^{-1} \mathcal{V}$ is a chainable covering of a space $X$.

Proposition 2.5. A Haudsorff space $X$ is arc-like if and only if each open covering $\mathcal{U}$ of $X$ admits a chainable refinement.

Proof. Assume that $X$ is arc-like and take an arbitrary open covering $\mathcal{U}$ of $X$. Then there exist an open covering $\mathcal{V}$ of $I$ and a surjection $f: X \rightarrow I$ such that $f^{-1} \mathcal{V}$ refines $\mathcal{U}$. Put $I=|K|$, where $K$ is a simplicial complex having two vertices 0,1 and one 1 -simplex. Then there exists a subdivision $L$ of $K$ such that a finite open covering $\mathcal{S}$ of $I$ consisting of open stars $\operatorname{st}(v, L)$ of the vertices $v$ of $L$ refines $\mathcal{V}$ (see Theorem 4 in [7, App. 1, §1.1]). Assume that the vertices $v_{i}, i=1, \ldots, n$, of $L$ are indexed in such a way that $0=v_{1}<$ $v_{2}<\cdots<v_{n-1}<v_{n}=1$ and put $W_{i}=\operatorname{st}\left(v_{i}, L\right), i=1, \ldots, n$. Note that $W_{i} \cap W_{j} \neq \emptyset$ if and only if vertices $v_{i}, v_{j}$ span a simplex of $L$. Consequently, $W_{i} \cap W_{j} \neq \emptyset$ if and only if $|i-j| \leq 1$, which shows that $\mathcal{S}=\left(W_{i}, i=1, \ldots, n\right)$ is a chainable covering of $I$. Then, $f^{-1} \mathcal{S}=\left(f^{-1} W_{i}, i=1, \ldots, n\right)$ is a chainable covering of $X$ which refines $f^{-1} \mathcal{V}$ and then also $\mathcal{U}$.

Conversely, assume that each open covering of $X$ admits a chainable refinement. First note that $X$ is compact and connected. Compactness is obvious since chainable coverings are open and finite by definition. Assume that $X$ is not connected. Then there exists an open covering $\mathcal{U}=\left(U_{1}, U_{2}\right)$ of 
$X$ consisting of two disjoint non-empty open sets. Let $\mathcal{V}=\left(V_{i}, i=1, \ldots, n\right)$ be a chainable refinement of $\mathcal{U}$. Since $V_{i} \cap V_{i+1} \neq \emptyset$, for each $i=1, \ldots, n-1$, it follows that all $V_{i}$ are contained in $U_{1}$ or all $V_{i}$ are contained in $U_{2 .}$. This contradicts the fact that $U_{1}$ and $U_{2}$ are both non-empty sets. Let us show that $X$ is arc-like. Take an open covering $\mathcal{U}$ of $X$ consisting of open sets $U \neq X$. Let $\mathcal{V}=\left(V_{i}, i=1, \ldots, n\right)$ be a chainable refinement of $\mathcal{U}$ and consider a canonical map $p_{\mathcal{V}}: X \rightarrow|N(\mathcal{V})|$ for $\mathcal{V}$, i.e. a map having property that $p_{\mathcal{V}}^{-1}(s t(V, N(\mathcal{V})) \subseteq V$, for each $V \in \mathcal{V}$ (see [7, page 326]). Since $|N(\mathcal{V})|$ is homeomorphic to $I, P=p_{\mathcal{V}}(X)$ is a compact and connected subset of $|N(\mathcal{V})|$ and $P$ is not a singleton, it follows that $P$ is homeomorphic to $I$ as well. Then there exist $j, k, 1 \leq j \leq k \leq n$, such that $\mathcal{W}=\left(\operatorname{st}\left(V_{i}, N(\mathcal{V})\right) \cap P, i=\right.$ $j, \ldots, k)$ is an open covering of $P$ consisting of non-empty sets. Note that $p_{\mathcal{V}}^{-1}\left(\operatorname{st}\left(V_{i}, N(\mathcal{V})\right) \cap P\right) \subseteq p_{\mathcal{V}}^{-1}\left(s t\left(V_{i}, N(\mathcal{V})\right)\right) \subseteq V_{i}, i=j, \ldots, k$, which shows that $p_{\mathcal{V}}^{-1}(\mathcal{W})$ refines $\mathcal{V}$ and also $\mathcal{U}$. Let $h: P \rightarrow I$ be a homeomorphism. Then $h p_{\mathcal{V}}: X \rightarrow I$ is a surjection, $\mathcal{W}^{\prime}=(h(W), W \in \mathcal{W})$ is an open covering of $I$ and $(h p \mathcal{V})^{-1}\left(\mathcal{W}^{\prime}\right)$ refines $\mathcal{U}$, which shows that $X$ is arc-like.

Definition 2.6. A Hausdorrf space $X$ is said to be chainable, if each open covering of $X$ admits a chainable refinement.

According to Proposition 2.5, a Hausdorff space $X$ is arc-like if and only if $X$ is chainable.

Definition 2.7. A covering $\left(A_{\lambda}, \lambda \in \Lambda\right)$ of a set $X$ is called irreducible if, for each $\lambda_{0} \in \Lambda$, a family $\left(A_{\lambda}, \lambda \in \Lambda \backslash\left\{\lambda_{0}\right\}\right)$ is not a covering of $X$. A covering $\left(A_{\lambda}, \lambda \in \Lambda\right)$ of a set $X$ is called reducible if it is not irreducible.

Put $I=|K|$,where $K$ is a simplicial complex consisting of two vertices 0,1 and one 1 -simplex. Let $L$ be a subdivision of $K$ with $n$ vertices $\left\{v_{1}, \ldots, v_{n}\right\}$ such that $0=v_{1}<v_{2}<\cdots<v_{n}=1$. Then an open covering $\mathcal{S}=\left(s t\left(v_{i}, L\right), i=1, \ldots, n\right)$ of $I$ consisting of open stars $s t\left(v_{i}, L\right)$ of the vertices $v_{i}$ of $L$ is an irreducible chainable covering of $I$. If $\mathcal{V}$ is an irreducible covering of a set $Y$ and $f: X \rightarrow Y$ is a surjective function, then $f^{-1} \mathcal{V}$ is an irreducible covering of a set $X$.

Lemma 2.8. Let $\left(A_{\lambda}, \lambda \in \Lambda\right)$ be an irreducible covering of a set $X$. Then for each $\lambda \in \Lambda$ there is an element $x_{\lambda} \in X$ such that $x_{\lambda} \in A_{\lambda}$ and $x_{\lambda} \notin A_{\lambda^{\prime}}$ for $\lambda^{\prime} \neq \lambda$.

Proof. Since $\left(A_{\lambda}, \lambda \in \Lambda\right)$ is an irreducible covering of $X$, for each $\lambda \in \Lambda$, a subset $X \backslash\left(\bigcup_{\lambda^{\prime} \neq \lambda} A_{\lambda^{\prime}}\right)$ of $X$ is non-empty and we can choose $x_{\lambda} \in X$ with the required properties.

Lemma 2.9. Let $\mathcal{U}=\left(U_{i}, i=1, \ldots, n\right), n \geq 2$, be a chainable covering of a connected space $X$. Then there exists an irreducible chainable subcovering $\mathcal{V}$ of $\mathcal{U}$. 
Proof. If $\mathcal{U}$ is irreducible, put $\mathcal{V}=\mathcal{U}$. Assume that $\mathcal{U}$ is reducible. If $n=2$, then $U_{1} \subseteq U_{2}$ or $U_{2} \subseteq U_{1}$. Putting $V_{1}=U_{2}$ in the first case or $V_{1}=U_{1}$ in the second case we get the desired subcovering $\mathcal{V}$. If $n>2$, then there exists $i \in\{1, \ldots, n\}$ such that $X=\bigcup_{j \neq i} U_{j}$.and we claim that $i \notin\{2, \ldots, n-1\}$. Indeed, assume the contrary. Then $X=\left(U_{1} \cup \cdots \cup U_{i-1}\right) \cup\left(U_{i+1} \cup \cdots \cup U_{n}\right)$ is the union of two non-empty disjoint open sets, which contradicts connectedness of $X$. Hence $i=1$ or $i=n$ and the desired irreducible chainable subcovering $\mathcal{V}$ of $\mathcal{U}$ is one of the coverings $\left(U_{i}, i=2, \ldots, n\right),\left(U_{i}, i=1, \ldots, n-1\right)$ or $\left(U_{i}, i=2, \ldots, n-1\right)$, depending on $i$.

Lemma 2.10. Let a Hausdorff space $X$ be arc-like. Then each open covering $\mathcal{U}$ of $X$ admits an irreducible chainable refinement $\mathcal{V}$.

Proof. The claim follows directly from the Proposition 2.5 and Lemma 2.9 .

Lemma 2.11. Let a Hausdorff space $X$ be arc-like and let $\left\{x_{1}, \ldots, x_{m}\right\}$ be a finite subset of $X$ consisting of different points. Then each open covering $\mathcal{U}$ of $X$ admits an irreducible chainable refinement $\mathcal{V}$ such that each point $x_{i}, i=1, \ldots, m$, belongs to exactly one member of $\mathcal{V}$ and different points $x_{i}, x_{j}, 1 \leq i, j \leq m$, belong to different members of $\mathcal{V}$.

Proof. Take an arbitrary open covering $\mathcal{U}$ of $X$. For each $i=1, \ldots, m$, put $W_{i}:=X \backslash\left\{x_{1}, \ldots, x_{i-1}, x_{i+1}, \ldots, x_{m}\right\}$. Then $\mathcal{W}=\left(W_{i}, i=1, \ldots, m\right)$ is an open covering of $X$ and, for each $i=1, \ldots, m, W_{i} \cap\left\{x_{1}, \ldots, x_{m}\right\}=\left\{x_{i}\right\}$. Let $\mathcal{U}^{\prime}$ be an open covering of $X$ which refines both $\mathcal{U}$ and $\mathcal{W}$. Since $X$ is an arc-like space there is a surjective map $f: X \rightarrow I$ and an open covering $\mathcal{V}^{\prime}$ of $I$ such that $f^{-1} \mathcal{V}^{\prime}$ refines $\mathcal{U}^{\prime}$. Note that $f\left(x_{i}\right) \neq f\left(x_{j}\right)$ for each $i \neq j$. Indeed, assume $f\left(x_{i}\right)=f\left(x_{j}\right)$, for some $i \neq j$. Take a $V^{\prime} \in \mathcal{V}^{\prime}$ such that $f\left(x_{i}\right)=f\left(x_{j}\right) \in V^{\prime}$ and let $W_{k}$ be an element of $\mathcal{W}$ with $f^{-1} V^{\prime} \subseteq W_{k}$. Then $x_{i}, x_{j} \in X \backslash\left\{x_{1}, \ldots, x_{k-1}, x_{k+1}, \ldots, x_{m}\right\}$, which implies $x_{i}=x_{j}=x_{k}$, and we get a contradiction. Consider $I$ as the carrier of a simplicial complex $K$ having two vertices 0,1 and one 1-simplex. Then there is a subdivision $L$ of $K$ such that a finite open covering $\mathcal{S}$ of $I$ consisting of open stars $s t(v, L)$ of the vertices $v$ of $L$ refines $\mathcal{V}^{\prime}$. Let $L^{\prime}$ be a subdivision of $L$ such that the set of vertices of $L^{\prime}$ contains all vertices of $L$ and all points $f\left(x_{i}\right), i=1, \ldots, m$. Denote by $\left\{v_{1}, \ldots, v_{n}\right\}, n \geq m$, the set of vertices of $L^{\prime}$ such that $0=v_{1}<$ $v_{2}<\cdots<v_{n}=1$. An open finite covering $\mathcal{S}^{\prime}$ of $I$ consisting of open stars $s t\left(v_{i}, L^{\prime}\right)$ of the vertices $v_{i}$ of $L^{\prime}$ refines $\mathcal{S}$ and also $\mathcal{V}^{\prime}$. Then $\mathcal{V}=f^{-1} \mathcal{S}^{\prime}$ is an irreducible chainable covering having required properties.

Let $\preceq$ be a binary relation on a set $\Lambda$. A subset $\Lambda^{\prime} \subseteq \Lambda$ is said to be cofinal in $\Lambda$, if, for each $\lambda \in \Lambda$, there exists some $\lambda^{\prime} \in \Lambda^{\prime}$ such that $\lambda \preceq \lambda^{\prime}$. If $(\Lambda, \preceq)$ is a directed preordered set and $\Lambda^{\prime} \subseteq \Lambda$ is cofinal in $\Lambda$, then $\left(\Lambda^{\prime}, \preceq\right)$ is a directed preordered set as well. Let $X$ be an arc-like space $X$ and denote by $\mathcal{I C}(X)$ 
a subset of $\operatorname{Cov}(X)$ consisting of all irreducible chainable coverings of $X$. According to Lemma $2.10, \mathcal{I C}(X)$ is cofinal in $(\operatorname{Cov}(X), \preceq)$ and, consequently, $(\mathcal{I C}(X), \preceq)$ is a directed preordered set.

THEOREM 2.12. Let a Hausdorff space $X$ be arc-like and let $\mathcal{I C}(X)$ be a subset of $\operatorname{Cov}(X)$ consisting of all irreducible chainable coverings of $X$. For each pair $\mathcal{U}, \mathcal{V} \in \mathcal{I C}(X)$ such that $\mathcal{U} \preceq \mathcal{V}$, select a projection puV : $|N(\mathcal{V})| \rightarrow$ $|N(\mathcal{U})|$. Then the obtained system $\left(|N(\mathcal{U})|, p_{\mathcal{U V}}, \mathcal{I C}(X)\right)$ is not an approximate system.

Proof. Assume the contrary, i.e. $\left(|N(\mathcal{U})|, p_{\mathcal{U V}}, \mathcal{I C}(X)\right)$ is an approximate system. Let $\mathcal{U} \in \mathcal{I C}(X)$ be an arbitrary index, i.e. $\mathcal{U}$ is an irreducible chainable covering of $X$ and assume $\mathcal{U}=\left(U_{1}, \ldots U_{n}\right), n \geq 2$. Choose an open covering $\mathcal{A}$ of $|N(\mathcal{U})|$ having property that each member of $\mathcal{A}$ contains at most one vertex $U \in|N(\mathcal{U})|$ (for instance, such $\mathcal{A}$ is an open covering $(\operatorname{st}(U, N(\mathcal{U})), U \in \mathcal{U})$ consisting of open stars of vertices $U$ of $N(\mathcal{U})$ ). Since the system $\left(|N(\mathcal{U})|, p_{\mathcal{U V}}, \mathcal{I C}(X)\right)$ is approximate, there exists a covering, i.e. an index, $\mathcal{U}_{0}=\left(U_{1}^{0}, \ldots, U_{m}^{0}\right) \in \mathcal{I C}(X)$ which refines $\mathcal{U}$, and for each $\mathcal{V}, \mathcal{W} \in \mathcal{I C}(X)$ satisfying $\mathcal{U}_{0} \preceq \mathcal{V} \preceq \mathcal{W}$, maps $p_{\mathcal{U} \mathcal{V}} p_{\mathcal{V} W}$ and $p_{\mathcal{U} \mathcal{W}}$ are $\mathcal{A}$-near. Take an arbitrary vertex $W \in|N(\mathcal{W})|$. Since the projections are determined by simplicial maps, points $p_{\mathcal{U} \mathcal{V}} p_{\mathcal{V W}}(W)$ and $p_{\mathcal{U} \mathcal{W}}(W)$ are vertices of the nerve $|N(\mathcal{U})|$. On the other hand, each member of $\mathcal{A}$ contains at most one vertex of $|N(\mathcal{U})|$, and we conclude that $p_{\mathcal{U V}} p_{\mathcal{V} \mathcal{W}}(W)=p_{\mathcal{U W}}(W)$, for each vertex $W \in|N(\mathcal{W})|$. This implies $p_{\mathcal{U} \mathcal{V}} p_{\mathcal{V W}}(y)=p_{\mathcal{U N}}(y)$, for each $y \in|N(\mathcal{W})|$. Thus, $p_{\mathcal{U} \mathcal{V}} p_{\mathcal{V W}}=p_{\mathcal{U} \mathcal{W}}$, for any $\mathcal{V}, \mathcal{W} \in \mathcal{I C}(X)$ such that $\mathcal{U}_{0} \preceq \mathcal{V} \preceq \mathcal{W}$. According to Lemma 2.8 , for each $i=1, \ldots, n$, we can choose a point $x_{i} \in X$ such that $x_{i} \in U_{i} \backslash\left(\bigcup_{j \neq i} U_{j}\right)$. Let $U_{i}^{0}$ and $U_{j}^{0}$ be members of the covering $\mathcal{U}_{0}$ with $x_{1} \in U_{i}^{0}$ and $x_{2} \in U_{j}^{0}$. Note that $i \neq j$. Indeed, assume that $i=j$. Since $U_{1}$ is the only member of $\mathcal{U}$ which contains $x_{1}$ and $U_{2}$ is the only member of $\mathcal{U}$ which contains $x_{2}$, we get $U_{i}^{0} \subseteq U_{1}$ and $U_{i}^{0}=U_{j}^{0} \subseteq U_{2}$. Hence, $x_{1} \in U_{1} \cap U_{2}$ and we get a contradiction. Hence, $i \neq j$. Note that $p_{\mathcal{U} \mathcal{U}_{0}}\left(U_{i}^{0}\right)=U_{1}$ and $p_{\mathcal{U} \mathcal{U}_{0}}\left(U_{j}^{0}\right)=U_{2}$. Without loss of generality assume $i<j$. We claim that there exists $k, i \leq k<j$, such that $\operatorname{pu\mathcal {U}}_{0}\left(U_{k}^{0}\right)=U_{1}$ and $p_{\mathcal{U} \mathcal{U}_{0}}\left(U_{k+1}^{0}\right)=U_{2}$. To prove that consider a finite set $F=\left\{n: \operatorname{pu\mathcal {U}}_{0}\left(U_{n}^{0}\right)=U_{1}, i \leq n<j\right\} . F$ is a non-empty set, since $i \in F$. Put $k:=\max F$. If $k=j-1$, the claim holds, because $p_{\mathcal{U} \mathcal{U}_{0}}\left(U_{j}^{0}\right)=U_{2}$. Assume $k<j-1$. Since $p_{\mathcal{U} \mathcal{U}_{0}}\left(U_{k}^{0}\right)=U_{1}$, it follows $U_{k}^{0} \subseteq U_{1} . \mathcal{U}_{0}$ is a chainable covering, so $U_{k}^{0} \cap U_{k+1}^{0} \neq \emptyset$. Let $U_{l} \in \mathcal{U}$ be a member of $\mathcal{U}$ such that $p_{\mathcal{U} \mathcal{U}_{0}}\left(U_{k+1}^{0}\right)=U_{l}$. Then $\emptyset \neq U_{k}^{0} \cap U_{k+1}^{0} \subseteq U_{1} \cap U_{l}$ and we conclude that $l \in\{1,2\}$. Assume that $p_{\mathcal{U} \mathcal{U}_{0}}\left(U_{k+1}^{0}\right)=U_{1}$. Since $i \leq k+1<j$, it follows that $k+1 \in F$ which contradicts $k=\max F$. Therefore, $\operatorname{puU}_{0}\left(U_{k+1}^{0}\right)=U_{2}$ and the claim is proved. So, we get $p_{\mathcal{U} \mathcal{U}_{0}}\left(U_{k}^{0}\right)=U_{1}$ and $\operatorname{puU}_{0}\left(U_{k+1}^{0}\right)=U_{2}$. Note that $U_{k}^{0} \cap U_{k+1}^{0}$ is an infinite set. Indeed, assume that $U_{k}^{0} \cap U_{k+1}^{0}$ is a finite set $U_{k}^{0} \cap U_{k+1}^{0}=\left\{y_{1}, y_{2}, \ldots, y_{n(k)}\right\}$. Then 
we get $\left\{y_{1}\right\}=\left(U_{k}^{0} \cap U_{k+1}^{0}\right) \backslash\left\{y_{2}, \ldots, y_{n(k)}\right\}$ which implies that $X$ has an isolated point $y_{1}$ and that contradicts connectedness of $X$. Choose arbitrary points $x, x^{\prime} \in U_{k}^{0} \cap U_{k+1}^{0}, x \neq x^{\prime}$. Let $\mathcal{V}_{1}$ and $\mathcal{V}_{2}$ be open coverings of $X$ given by $\mathcal{V}_{1}=\left(U_{1}^{0}, \ldots, U_{k-1}^{0}, U_{k}^{0} \backslash\{x\}, U_{k+1}^{0} \backslash\left\{x^{\prime}\right\}, U_{k+2}^{0}, \ldots, U_{m}^{0}\right), \mathcal{V}_{2}=$ $\left(U_{1}^{0}, \ldots, U_{k-1}^{0}, U_{k}^{0} \backslash\left\{x^{\prime}\right\}, U_{k+1}^{0} \backslash\{x\}, U_{k+2}^{0}, \ldots, U_{m}^{0}\right)$. Note that $\mathcal{V}_{1}$ and $\mathcal{V}_{2}$ are irreducible chainable coverings of $X$ which refine $\mathcal{U}_{0}$, i.e. $\mathcal{V}_{1}, \mathcal{V}_{2} \in \mathcal{I C}(X)$, $\mathcal{U}_{0} \preceq \mathcal{V}_{1}, \mathcal{V}_{2}$. Moreover, $p_{\mathcal{U}_{0} \mathcal{V}_{1}}\left(U_{k+1}^{0} \backslash\left\{x^{\prime}\right\}\right)=U_{k+1}^{0}$ and $p_{\mathcal{U}_{0} \mathcal{V}_{2}}\left(U_{k}^{0 \backslash} \backslash\left\{x^{\prime}\right\}\right)=$ $U_{k}^{0}$. According to Lemma 2.11, there exists an irreducible chainable covering $\mathcal{W}$ of $X$ which refines both $\mathcal{V}_{1}$ and $\mathcal{V}_{2}$, each of the points $x, x^{\prime}$ belongs to exactly one member of $\mathcal{W}$ and $x, x^{\prime}$ belong to different members of $\mathcal{W}$. Hence $\mathcal{W} \in \mathcal{I C}(X)$ and $\mathcal{U}_{0} \preceq \mathcal{V}_{1}, \mathcal{V}_{2} \preceq \mathcal{W}$. Let $W \in \mathcal{W}$ be the only element of $\mathcal{W}$ which contains $x$. Note that $x^{\prime} \notin W$ and $U_{k+1}^{0} \backslash\left\{x^{\prime}\right\}$ is the only element of $\mathcal{V}_{1}$ which contains $x$. This implies $p_{\mathcal{V}_{1} \mathcal{W}}(W)=U_{k+1}^{0} \backslash\left\{x^{\prime}\right\}$ and we get $p_{\mathcal{U} \mathcal{W}}(W)=p_{\mathcal{U} \mathcal{V}_{1}} p_{\mathcal{V}_{1} \mathcal{W}}(W)=p_{\mathcal{U} \mathcal{V}_{1}}\left(U_{k+1}^{0} \backslash\left\{x^{\prime}\right\}\right)=p_{\mathcal{U} \mathcal{U}_{0}} p_{\mathcal{U}_{0} \mathcal{V}_{1}}\left(U_{k+1}^{0} \backslash\left\{x^{\prime}\right\}\right)=$ $p_{\mathcal{U U}_{0}}\left(U_{k+1}^{0}\right)=U_{2}$. On the other hand, $U_{k}^{0} \backslash\left\{x^{\prime}\right\}$ is the only element of $\mathcal{V}_{2}$ which contains $x$ and we get $p_{\mathcal{U W}}(W)=p_{\mathcal{U} \mathcal{V}_{2}} p_{\mathcal{V}_{2} \mathcal{W}}(W)=p_{\mathcal{U} \mathcal{V}_{2}}\left(U_{k}^{0} \backslash\left\{x^{\prime}\right\}\right)=$ $p_{\mathcal{U} \mathcal{U}_{0}} p_{\mathcal{U}_{0} \mathcal{V}_{2}}\left(U_{k}^{0} \backslash\left\{x^{\prime}\right\}\right)=p_{\mathcal{U} \mathcal{U}_{0}}\left(U_{k}^{0}\right)=U_{1}$. This yields a contradiction since $U_{1}$ and $U_{2}$ are different vertices of $|N(\mathcal{U})|$.

TheOREM 2.13. The Čech system $\boldsymbol{C}(X)=\left(|N(\mathcal{U})|,\left[p_{\mathcal{U V}}\right], \operatorname{Cov}(X)\right)$ of a Hausdorff arc-like space $X$ does not induce approximate systems, i.e. it is not possible to select one projection $q_{\mathcal{U V}}$ in each homotopy class $\left[p_{\mathcal{U V}}\right], \mathcal{U} \preceq \mathcal{V}$, in such a way that $\left(|N(\mathcal{U})|, q_{\mathcal{U}}, \operatorname{Cov}(X)\right)$ becomes an approximate system.

Proof. Assume the contrary, i.e. there exists a selection of projections $q_{\mathcal{U V}} \in\left[p_{\mathcal{U V}}\right]$, for each pair of coverings $\mathcal{U} \preceq \mathcal{V}$, such that the obtained system $\left(|N(\mathcal{U})|, q_{\mathcal{U V}}, \operatorname{Cov}(X)\right)$ is an approximate system. Then $\left(|N(\mathcal{U})|, q_{\mathcal{U V}}, \mathcal{I C}(X)\right)$ is an approximate system, too, which contradicts Theorem 2.12.

\section{REFERENCES}

[1] S. Mardešić, Chainable continua and inverse limits, Glasnik Mat.-Fiz. Astronom. 14 (1959), 219-232.

[2] S. Mardešić, On covering dimension and inverse limits for compact spaces, Illinois J. Math. 4 (1960), 278-291.

[3] S. Mardešić and L. R. Rubin, Approximate inverse systems of compacta and covering dimension, Pacific J. Math. 138 (1989), 129-144.

[4] S. Mardešić and J. Segal, Shape Theory. The inverse system approach, North Holland, Amsterdam, 1982.

[5] S. Mardešić and T. Watanabe, Approximate resolutions of spaces and mappings, Glas. Mat. Ser. III 24 (1989), 587-637. 


\section{Čechov sustav ne inducira aproksimativne sustave}

\section{Vlasta Matijević}

SAŽETAK. Svakom topološkom prostoru $X$ pridružen je njegov Čechov sustav $C(X)=\left(|N(\mathcal{U})|,\left[p_{\mathcal{U V}}\right], \operatorname{Cov}(X)\right)$. Dobro je poznato da je Čechov sustav $C(X)$ od $X$ inverzni sustav u homotopskoj kategoriji $\mathrm{HPol}$ čiji su objekti poliedri, a morfizmi homotopske klase neprekidnih preslikavanja među poliedrima. Sibe Mardešić postavio je sljedeće pitanje: Za dani Čechov sustav $\left(|N(\mathcal{U})|,\left[p_{\mathcal{U V}}\right], \operatorname{Cov}(X)\right)$ prostora $X$, je li moguće izabrati član $q_{\mathcal{U V}} \in\left[p_{\mathcal{U V}}\right]$ u svakoj homotopskoj klasi $\left[p_{\mathcal{U V}}\right]$ tako da dobiveni sustav $\left(|N(\mathcal{U})|, q_{\mathcal{U V}}, \operatorname{Cov}(X)\right)$ bude aproksimativni sustav? Ovdje pokazujemo da je odgovor na to pitanje negativan, budući da, za svaki Hausdorffov kontinuum $X$ koji je poput luka, svaki takav sustav $\left(|N(\mathcal{U})|, q_{\mathcal{U V}}, \operatorname{Cov}(X)\right)$ nije aproksimativni sustav.

Vlasta Matijević

Department of Mathematics

Faculty of Science

University of Split

21000 Split, Croatia

E-mail: vlasta@pmfst.hr

Received: 31.1.2017.

Revised: 12.2.2017. 